\title{
A sofic system which is not spectrally of finite type
}

\author{
SUSAN WILLIAMS* \\ University of South Alabama, Mobile, Alabama 36688, USA \\ (Received 30 September 1986 and revised April 1987)
}

\begin{abstract}
We exhibit a transitive sofic system for which the core matrix has negative trace, and hence cannot share the nonzero spectrum of any subshift of finite type cover. We also show that every transitive sofic system has an integral core matrix.
\end{abstract}

\section{Introduction}

A sofic system is a symbolic dynamical system $S$ which is the image of some subshift of finite type $\Sigma$ under a continuous shift-commuting map; we call $\Sigma$ a cover of $S$. Nasu [N] defined the core matrix (a square matrix with real entries, uniquely determined up to similarity) of a transitive sofic system and showed that the block of the Jordan form of the core matrix with non-zero eigenvalues is an invariant for finite-to-one factor maps between transitive sofic systems. This in an extension of a result of Kitchens [K], that the Jordan form away from zero of the adjacency matrix of an irreducible subshift of finite type is an invariant for finite-to-one factor maps. Much research has been done on the question of what matrices can occur as Jordan forms of subshifts of finite type, and it is natural to ask the same question for core matrices. In particular, are the two classes the same?

A transitive sofic system is spectrally of finite type if there is a sofic system $S_{1}$ conjugate to $S$ and a cover $\Sigma_{1}$ of $S_{1}$ such that the adjacency matrix of $\Sigma_{1}$ is a core matrix of $S_{1}$. Then the core matrix of $S$ and the adjacency matrix of $\Sigma_{1}$ have the same Jordan form away from zero. Nasu showed that the class of spectrally finite type sofic systems properly contains the almost finite type systems (see $\S 2$ for a definition), and conjectured that every transitive sofic system is spectrally of finite type.

We present a counterexample to this conjecture. In fact, the core matrix in our example has negative trace, so its Jordan form away from zero cannot be that of any non-negative integral matrix. On the other hand, we show that every transitive sofic system has an integral core matrix.

In $\S \S 1$ and 2 we give a synopsis of basic definitions and results concerning matrix systems and the core. $\$ 3$ contains a construction of a rational core matrix system for any given transitive sofic system; from this we deduce the existence of an integral core matrix (Theorem 3.4). Under certain easily-checked conditions the construction

\footnotetext{
* Partially supported by grants from the University of South Alabama Research and Public Service Council and the University of South Alabama Research Committee.
} 
yields a non-negative integral matrix (Remark 3.3). This observation was the motivation for our example of a transitive sofic system which is not spectrally of finite type. The example is given in $\S 4$, and can be read independently by anyone familiar with Nasu's paper [N].

\section{Acknowledgements}

I am grateful to the referee for correcting the proof of Lemma 3.2, and to Mike Boyle for very helpful discussions. I thank the Mathematics Institute of the University of Warwick for its hospitality during the Summer of 1986.

1. Sofic systems, matrix systems and the core

Let $A$ be a finite set (alphabet). We denote by $A^{*}$ the set of finite strings (words) of elements of $A$, including the empty string $\Lambda$; a language is a subset of $A^{*}$.

A subshift $S$ of $A^{Z}$ is a closed subset which is invariant under the left shift map $\sigma ; S$ is completely determined by its language, that is, the set of words appearing in elements of $S . S$ is sofic if there is a finite semigroup $G$ with absorbing element $O$ (that is, $O g=g O=O$ for all $g \in G$ ) and a distinguished set of distinct generators $\{g(a): a \in A\}$ such that the language of $S$ is exactly the set of $x=x_{1} \cdots x_{n} \in A^{*}$ for which $g\left(x_{1}\right) \cdots g\left(x_{n}\right) \neq O$. We say $S$ is transitive if for every $x$ and $y$ in the language of $S$ there is a word $z$ with $x z y$ in the language of $S$. (Some authors use a weaker definition of transitive and refer to the above condition as 'transitive, periodic points dense'.) A factor map between subshifts is a continuous, onto shift-commuting map. Two subshifts are (topologically) conjugate if there is an invertible factor map between them.

A matrix system of order $m$ over $A$ consists of a triple $(A, M, \Phi)$ where $\Phi$ is a homomorphism of the monoid $A^{*}$ into the multiplicative monoid of $m \times m$ real matrices (which means $\Phi(\Lambda)=I_{m}$ and $\Phi(x)=\Phi\left(x_{1}\right) \cdots \Phi\left(x_{n}\right)$ for $x=x_{1} \cdots x_{n} \in A^{*}$ ) and $M=\sum_{a \in A} \Phi(a)$ is nondegenerate (that is, no row or column consists only of zeros). We say $(A, M, \Phi)$ is irreducible if $M$ is irreducible, and integral [respectively, non-negative] if each $\Phi(a)$ has integral [non-negative] entries. The language of a non-negative matrix system $(A, M, \Phi)$, denoted $L(A, M, \Phi)$, is the set of words $x \in A^{*}$ for which $\Phi(x) \neq 0$. Then $L(A, M, \Phi)$ is given by the semigroup $G=\Phi\left(A^{*}\right)$ with generators $\Phi(a)$, and determines a sofic subshift.

A non-negative integral matrix system $(A, M, \Phi)$ corresponds in a natural way to a labelled directed graph. We let $G(M)$ be the graph with vertices (states) $1, \ldots, m$, where $m$ is the order of $(A, M, \Phi)$, and $M_{i j}$ edges from state $i$ to state $j,(\Phi(a))_{i j}$ of which are labelled with the symbol $a$. Let $E$ be the edge set of $G(M)$; we say an edge $f$ follows the edge $e$ if the initial state of $f$ is the terminal state of $e$. The subshift of finite type (SFT) $\Sigma_{M}$ is the subshift

$$
\left\{\left(e_{i}\right) \in E^{Z}: e_{i+1} \text { follows } e_{i} \text { for all } i\right\} .
$$

The sofic system $S$ with language $L(A, M, \Phi)$ is simply the image of $\Sigma_{M}$ under the map $\pi: E^{Z} \rightarrow A^{Z}$ which sends each coordinate edge to its label. It is well known (cf. [CP]) that every sofic system is the image of some subshift of finite type under such a '1-block' map. We will refer to $\left(\Sigma_{M}, \pi\right)$ - or sometimes just $\Sigma_{M}$ - as the 
(SFT) cover of $S$ corresponding to the non-negative integral matrix system $(A, M, \Phi)$. A sofic system is transitive if and only if it has a cover $\Sigma_{M}$ with $M$ irreducible [F, CP].

A non-negative matrix system $(A, M, \Phi)$ is said to be normal if there are constants $0<c<d$ such that

$$
c<\sum_{i, j}(\Phi(x))_{i j}<d \quad \text { for all } x \in L(A, M, \Phi) .
$$

In the integral case $(\Phi(x))_{i j}$ is the number of paths in $G(M)$ beginning at state $i$ and ending at $j$ which are labeled by the word $x$; thus an irreducible matrix system is normal if and only if the cover map $\pi$ is finite-to-one.

We sometimes consider $(A, M, \Phi)$ with specified initial and final vectors $(u, v)$, where $u$ and $v$ are $m$-dimensional row, respectively column vectors. $((A, M, \Phi)$ with $(u, v)$ is also called a linear space automaton [MIF, IFM]). We say $(A, M, \Phi)$ with $(u, v)$ is equivalent to $(A, N, \Psi)$ with $\left(u^{\prime}, v^{\prime}\right)$ if

$$
u \Phi(x) v=u^{\prime} \Psi(x) v^{\prime}, \quad \text { all } x \in A^{*} \text {. }
$$

If $(A, M, \Phi)$ is non-negative and irreducible then $M$ has a positive maximal eigenvalue $\lambda_{M}$ and corresponding positive left, respectively right eigenvectors $u_{M}, v_{M}$ with $\left\|u_{M}\right\|=1, u_{M} v_{M}=1$. Then $\Phi(x)=0$ if and only if $u_{M} \Phi(x) v_{M}=0$. In particular, if $(A, N, \Psi)$ is also non-negative and irreducible and $(A, M, \Phi)$ with $\left(u_{M}, v_{M}\right)$ is equivalent to $(A, N, \Psi)$ with $\left(u_{N}, v_{N}\right)$ then $L(A, M, \Phi)=L(A, N, \Psi)$. Conversely,

THEOREM A [N]. If $(A, M, \Phi)$ and $(A, N, \Psi)$ are normal irreducible non-negative matrix systems with the same language then $\lambda_{M}=\lambda_{N}$ and $(A, M, \Phi)$ with $\left(u_{M}, v_{M}\right)$ is equivalent to $(A, N, \Psi)$ with $\left(u_{N}, v_{N}\right)$.

The matrix system $(A, M, \Phi)$ with $(u, v)$ is said to be row reduced if $\left\{u \Phi(x): x \in A^{*}\right\}$ generates the row vector space $R^{m}$, column reduced if $\left\{\Phi(x) v: x \in A^{*}\right\}$ generates the column space $R^{m}$, and reduced if both these conditions hold. By a reduced form of $(A, M, \Phi)$ with $(u, v)$ we mean an equivalent system which is reduced. Reduced forms always exist; constructions appear in [IFM, N]. In $\S 2$ we give a variant of the latter.

We say two matrix systems $(A, M, \Phi)$ and $(A, N, \Psi)$ are similar if they are of the same order and there is a non-singular matrix $T$ with $\Phi(a) T=T \Psi(a)$ for all $a \in A$. In this case we see that $(A, M, \Phi)$ with $(u, v)$ is equivalent to $(A, N, \Psi)$ with $\left(u T, T^{-1} v\right)$. For reduced forms we have the converse:

THEOREM B [N]. If $(A, M, \Phi)$ with $(u, v)$ and $(A, N, \Psi)$ with $\left(u^{\prime}, v^{\prime}\right)$ are reduced and equivalent then $(A, M, \Phi)$ and $(A, N, \Psi)$ are similar.

Corollary. If $(A, \bar{M}, \bar{\Phi})$ with $(\bar{u}, \bar{v})$ is a reduced form of $(A, M, \Phi)$ with $(u, v)$ then the Jordan form of $\bar{M}$ is a principal submatrix of the Jordan form of $M$. If $u, v$ are eigenvectors for an eigenvalue $\lambda$ of $M$ then $\lambda$ is also an eigenvalue of $\bar{M}$ with eigenvectors $\bar{u}, \bar{v}$.

Proof. This is easily checked for the reduced form given by the construction. Since these properties are preserved under similarity, they hold for any reduced form. 
Definition [N]. For a transitive sofic system $S$, let $(A, M, \Phi)$ be a normal irreducible non-negative matrix system with language equal to the language of $S$, and let $(A, \bar{M}, \bar{\Phi})$ with $(\bar{u}, \bar{v})$ be a reduced form of $(A, M, \Phi)$ with $\left(u_{M}, v_{M}\right)$. Then we call $(A, \bar{M}, \bar{\Phi})$ a core matrix system for $S$, and $\bar{M}$ a core matrix of $S$.

By Theorems $A$ and $B$, any two core matrix systems for $S$ are similar. By the corollary, $\lambda_{M}$ is an eigenvalue of $\bar{M}$ - in fact, its maximal eigenvalue - and $\bar{u}$ and $\bar{v}$ are corresponding eigenvectors. Hence when we speak of a core matrix system $(A, \bar{M}, \bar{\Phi})$ there is no need to specify initial and final vectors: we can retrieve the language of $S$ as

$$
\left\{x \in A^{*}: u_{\bar{M}} \bar{\Phi}(x) v_{\bar{M}} \neq 0\right\} .
$$

Since the core matrix system involves the alphabet $A$ of $S$ it is by no means invariant under topological conjugacy. The core matrix, however, provides an invariant:

THEOREM C [N]. If the transitive sofic system $S$ is a factor of the transitive sofic system $T$ then the Jordan form away from zero of the core matrix $S$ is a principal submatrix of the Jordan form of the core matrix of $T$. Thus the Jordan form away from zero of the core matrix is an invariant of topological conjugacy.

\section{Nasu's conjecture}

The SFT cover of a sofic system corresponding to a non-negative integral matrix system $(A, M, \Phi)$ is said to be right [left] resolving if each $\Phi(a)$ is a $0-1$ matrix with at most one 1 in each row [column]. For a given transitive sofic $S$, a well-known construction [F] yields a minimal right resolving cover $R$ and a minimal left resolving cover $L$.

THEOREM $\mathrm{D}[\mathrm{N}]$. If the integral non-negative matrix system $(A, M, \Phi)$ corresponds to the minimal right $[$ left $]$ resolving cover of a transitive sofic system $S$ then $(A, M, \Phi)$ with $\left(u_{M}, v_{M}\right)$ is row [column] reduced.

A sofic system $S$ is said to be of almost finite type (AFT) if it has an irreducible SFT cover $(\Sigma, \pi)$ such that $\pi$ is one-to-one on a non-trivial open set [M]. Equivalently (see [N, BKM]), $S$ is topologically conjugate to a transitive sofic system $S_{1}$ for which the minimal right resolving and minimal left resolving covers are the same. Then the corresponding matrix system $(A, N, \Psi)$ is row and column reduced; that is, it is a core matrix system for $S_{1}$. In particular, $N$ and the core matrix of $S$ have the same Jordan form away from zero.

If $S$ is not of almost finite type it can happen that the core matrix system is smaller than the matrix systems corresponding to $R$ and $L$, not only in order, but in the spectral sense that the matrices of $R$ and $L$ have non-zero eigenvalues which are not eigenvalues of the core matrix. Nasu's paper contains such a 'small core' example. In Nasu's example, however, the similarity class of the core matrix contains a non-negative integral matrix system, which therefore corresponds to an SFT cover of $S$. 
Definition [N]. A transitive sofic system $S$ is spectrally of finite type if there exist a sofic system $S_{1}$ topologically conjugate to $S$ and a 1-block SFT cover $\Sigma_{1}$ of $S_{1}$ such that the matrix system corresponding to the cover is a core matrix system for $S_{1}$.

Thus all AFT sofic systems as well as Nasu's non-AFT 'small core' example are spectrally of finite type. Nasu conjectured that every sofic system is spectrally of finite type. It is this conjecture which we disprove in $\S 4$.

This negative result leaves open the common factors problem: do two sofic systems $S, T$ of equal entropy necessarily have a common equal-entropy sofic factor? If 'sofic' is replaced by 'SFT' the answer is no [K]; one way to produce a counterexample is to construct $S$ and $T$ so that the only common principal submatrix of their rational canonical forms away from zero has negative trace (cf. [L]). If Nasu's conjecture were true we could conclude as well that $S$ and $T$ have no common equal-entropy sofic factor.

\section{A construction of the core}

We give a practical method of constructing the core matrix system for a transitive sofic system $S$. Our construction is a special case of Nasu's general method of obtaining a reduced form; by making more explicit choices at several places in the construction we ensure that the core matrix system we obtain has only rational entries. From this we show that there is an integral core matrix.

Construction 3.1. Let $(A, M, \Phi)$ be the matrix system corresponding to the minimal right resolving cover of $S$. By Theorem $\mathrm{D},(A, M, \Phi)$ with $\left(u_{M}, v_{M}\right)$ is row reduced. To column reduce it (if it is not already reduced) we must first choose a basis for the column vector space

$$
V=\left\{\Phi(x) v_{M}: x \in A^{*}\right\}
$$

We say a word $x \in A^{*}$ is resolving for the minimal right resolving cover if all paths labelled with $x$ in the graph $G(M)$ of the cover end at the same state. Resolving words exist [F], and every extension of a resolving word is resolving. If $x$ is resolving, we denote by $1_{x}$ the column vector in $R^{m}$ with a 1 in the $i$ th coordinate if some appearance of $x$ in $G(M)$ begins at state $i$, and 0 otherwise.

\section{LeMMA 3.2. Let $P=\left\{1_{x}: x\right.$ a resolving word $\}$. Then $P$ spans $V$.}

Proof. For any word $x$ of $S$, the $i j$ coordinate of $\Phi(x)$ is simply the number of paths in $G(M)$ from state $i$ to state $j$ which are labelled with the word $x$; this number will be 0 or 1 since a right resolving cover is finite-to-one. If $x$ is resolving, with all appearances ending at state $j$, then $\Phi(x) v_{M}=\left(v_{M}\right)_{j} 1_{x}$.

If $x$ is not resolving, fix a resolving word $z$ and let $W$ be the set of words $w$ such that $x w$ is a word of $S, w$ ends in $z$ and $z$ appears nowhere else in $w$. For every word $s \in S$, the measure of maximal entropy on $S$ (see $[\mathbf{P}]$ ) assigns to the cylinder set determined by $s$ the mass

$$
m(s)=\lambda_{M}{ }^{-|s|} u_{M} \Phi(s) v_{M},
$$

where $|s|$ is the length of $s$. Then $m(s x)=p q=p r$, where

$$
\begin{gathered}
p=\lambda_{M}{ }^{-|s|} u_{M} \Phi(s), \quad q=\lambda_{M}{ }^{-|x|} \Phi(x) v_{M}, \\
r=\sum_{w \in W} \lambda_{M}{ }^{-|x w|} \Phi(x w) v_{M} .
\end{gathered}
$$


Since $(A, M, \Phi)$ is row reduced, the vectors $p$ generate $R^{m}$ as $s$ ranges through the words of $S$. Thus $q$ and $r$ are identical as linear functionals on $R$, so $q=r$. But we have seen that each $\Phi(x w) v_{M}$ is in the span of $P$, and so $r$ is as well since finite-dimensional vector spaces are closed.

From $P$ we choose a basis for $V$, and form a 0-1 matrix $K$ which has these basis elements as columns. Then for each $a \in A$ there is a unique $\bar{m} \times \bar{m}$ matrix $\bar{\Phi}(a)$, where $\bar{m}$ is the dimension of $V$, satisfying

$$
\Phi(a) K=K \bar{\Phi}(a)
$$

and the sum $\bar{M}$ of these matrices satisfies $M K=K \bar{M}$. Let $\bar{v}$ be the $\bar{m}$-dimensional column vector with $v_{M}=K \bar{v}$, and $\bar{u}=u_{M} K$. Then $(A, \bar{M}, \bar{\Phi})$ with $(\bar{u}, \bar{v})$ is reduced and equivalent to $(A, M, \Phi)$ with $\left(u_{M}, v_{M}\right)$ (see $[\mathrm{N}$, p. 95$]$ for details), so it is a core matrix system for $S$. Since $\Phi(a)$ and $K$ are integral matrices, it follows from (*) that $\bar{\Phi}(a)$ is a rational matrix for every $a$, and $\bar{M}$ is rational as well.

Remark 3.3. If in the above construction it is possible to choose the basis of $V$ from $P$ so that every element of $P$ is a sum of basis elements, then the resulting core matrix system will be integral and non-negative, and so $S$ is spectrally of finite type. In Nasu's small core example,

$$
P=\left\{(1000)^{t},(0101)^{t},(0011)^{t},(1101)^{t},(1011)^{t}\right\}
$$

and so the first three elements form a basis of the desired type. The motivation of our example in $\$ 4$ was to produce a sofic system for which no such basis exists. This by itself does not guarantee that the system is not spectrally of finite type, but it provides a good starting point for further experimentation.

THEOREM 3.4. Every transitive sofic system has an integral core matrix.

Proof. By Construction 3.1 we can find a rational core matrix $\bar{M}$. The Jordan form away from zero of $\bar{M}$ is a principal submatrix of the Jordan form of the matrix $M$ of an equal-entropy SFT cover. Hence the characteristic polynomial $\bar{p}(t)$ of $\bar{M}$ divides a power of $t$ times the characteristic polynomial $p(t)$ of $M$. Since $\bar{p}(t)$ has rational coefficients, $p(t)$ integral coefficients, and both are monic, it follows from the Gauss Lemma that $\bar{p}(t)$ has integral coefficients. Then the companion matrix of $\bar{p}(t)$ is an integral matrix similar to $\bar{M}$, so it is an integral core matrix for the given sofic system.

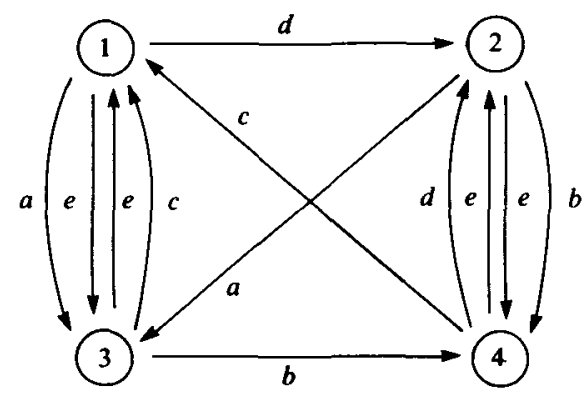

FIGURE 1 


\section{The example}

Let $S$ be the sofic system given by the graph in figure 1 . The cover shown corresponds to the matrix system

$$
\begin{array}{ccc}
\Phi(a)=\left(\begin{array}{llll}
0 & 0 & 1 & 0 \\
0 & 0 & 1 & 0 \\
0 & 0 & 0 & 0 \\
0 & 0 & 0 & 0
\end{array}\right), & \Phi(b)=\left(\begin{array}{llll}
0 & 0 & 0 & 0 \\
0 & 0 & 0 & 1 \\
0 & 0 & 0 & 1 \\
0 & 0 & 0 & 0
\end{array}\right), & \Phi(c)=\left(\begin{array}{llll}
0 & 0 & 0 & 0 \\
0 & 0 & 0 & 0 \\
1 & 0 & 0 & 0 \\
1 & 0 & 0 & 0
\end{array}\right) \\
\Phi(d)=\left(\begin{array}{llll}
0 & 1 & 0 & 0 \\
0 & 0 & 0 & 0 \\
0 & 0 & 0 & 0 \\
0 & 1 & 0 & 0
\end{array}\right), & \Phi(e)=\left(\begin{array}{llll}
0 & 0 & 1 & 0 \\
0 & 0 & 0 & 1 \\
1 & 0 & 0 & 0 \\
0 & 1 & 0 & 0
\end{array}\right), & M=\left(\begin{array}{llll}
0 & 1 & 2 & 0 \\
0 & 0 & 1 & 2 \\
2 & 0 & 0 & 1 \\
1 & 2 & 0 & 0
\end{array}\right)
\end{array}
$$

Carrying out Construction 3.1 we find

$$
P=\left\{(1100)^{t},(0110)^{t},(0011)^{t},(1001)^{t}\right\},
$$

so that no choice of basis meets the condition of Remark 3.3. Taking

$$
K=\left(\begin{array}{lll}
1 & 0 & 0 \\
1 & 1 & 0 \\
0 & 1 & 1 \\
0 & 0 & 1
\end{array}\right)
$$

we find the core matrix system

$$
\begin{array}{ccc}
\bar{\Phi}(a)=\left(\begin{array}{rrr}
0 & 1 & 1 \\
0 & 0 & 0 \\
0 & 0 & 0
\end{array}\right), & \bar{\Phi}(b)=\left(\begin{array}{rrr}
0 & 0 & 0 \\
0 & 0 & 1 \\
0 & 0 & 0
\end{array}\right), & \bar{\Phi}(c)=\left(\begin{array}{rrr}
0 & 0 & 0 \\
0 & 0 & 0 \\
1 & 0 & 0
\end{array}\right) \\
\bar{\Phi}(d)=\left(\begin{array}{rrr}
1 & 1 & 0 \\
-1 & -1 & 0 \\
1 & 1 & 0
\end{array}\right), & \bar{\Phi}(e)=\left(\begin{array}{rrr}
0 & 1 & 1 \\
0 & -1 & 0 \\
1 & 1 & 0
\end{array}\right), & \bar{M}=\left(\begin{array}{rrr}
1 & 3 & 2 \\
-1 & -2 & 1 \\
3 & 2 & 0
\end{array}\right) .
\end{array}
$$

Note that the core matrix $\bar{M}$ has negative trace. Any matrix having the same Jordan form away from zero as $\bar{M}$ will also have negative trace, so it must have negative entries. Since the Jordan form away from zero of the core matrix is a conjugacy invariant, no sofic system topologically conjugate to $S$ has a core matrix system corresponding to $a$ an SFT cover. Hence $S$ is not spectrally of finite type.

\section{REFERENCES}

[AM] R. Adler \& B. Marcus. Topological entropy and equivalence of dynamical systems. Mem. AMS 219 (1979).

[BKM] M. Boyle, B. Kitchens \& B. Marcus. A note on minimal covers for sofic systems. Proc. AMS 95 (1985), 403-411.

[CP] E. Coven and M. Paul, Sofic systems. Israel J. Math. 20 (1975), 165-177.

[F] R. Fischer. Sofic systems and graphs. Monatsh. Math. 80 (1975), 179-186.

[IFM] Y. Inagaki, T. Fukumura \& H. Matuura. Some aspects of linear space automata. Inform. and Contr. 20 (1972), 439-479.

[K] B. Kitchens. An invariant for continuous factors of Markov shifts. Proc. AMS 83 (1981), 825-828. 
[L] D. Lind. The spectra of topological Markov chains. Ergod. Th. \& Dynam. Sys. 6(1986), 571-582.

[M] B. Marcus. Sofic systems and encoding data. IEEE Trans. Information Theory 31 (1985), 366-377.

[MIF] H. Matuura, Y. Inagaki \& T. Fukumura. A generalization of automata and its analysis. Records Natl. Conv. IECE, Japan A67-45 (1968) (in Japanese).

[N] M. Nasu. An invariant for bounded-to-one factor maps between transitive sofic subshifts. Ergod. Th. \& Dynam. Sys. 5 (1985), 89-105.

[P] W. Parry. Intrinsic Markov chains. Trans. AMS 112 (1964), 55-66.

[W] B. Weiss. Subshifts of finite type and sofic systems. Monatsh. Math. 77 (1973), 462-474. 